\title{
Radiographic changes of the patellar ligament in dogs after tibial tuberosity advancement
}

\author{
Ladislav Stehlík ${ }^{1}$, Pavel Proks ${ }^{1}$, Petra Fedorová ${ }^{2}$, Alois Nečas ${ }^{2}$
}

${ }^{1}$ University of Veterinary and Pharmaceutical Sciences Brno, Faculty of Veterinary Medicine, Small Animal Clinic, Department of Diagnostic Imaging, ${ }^{2}$ Department of Surgery and Orthopaedics, Brno, Czech Republic

\author{
Received September 16, 2012 \\ Accepted February 19, 2013
}

\begin{abstract}
Patellar desmopathy in dogs after tibial plateau levelling osteotomy has been described in many studies. Tibial tuberosity advancement is a biomechanically different technique. It is assumed that the patellar ligament is loaded with little force similarly as after tibial plateau levelling osteotomy. Various aspects related to secondary patellar desmopathy are not completely understood. This study deals with computed radiography measurement of patellar ligament thickness after tibial tuberosity advancement in dogs with cranial cruciate ligament rupture. The thickness of the patellar ligament in exactly predetermined locations was measured from mediolateral radiographs of stifle joints. A total of 18 dogs (20 knee joints) with cranial cruciate ligament (ligamentum cruciatum craniale) rupture underwent three radiographic examinations of the knee (preoperative examination and control examination 7 and 15 weeks after the surgery). Significant difference was found between the thickness of the patellar ligament in the first and second examinations. Some of the demographic factors possibly related to patellar ligament thickness (age, sex, body weight, type of cranial cruciate ligament rupture, arthrotomy, cage size, meniscal injury, time between surgery and radiographic examination) were analyzed. However, statistical analyses did not show any effect of these factors on the thickness of the patellar ligament, except for the time between surgery and radiographic examination. These findings can extend the surgeons' knowledge of biomechanical aspects of tibial tuberosity advancement.
\end{abstract}

Computed radiography, ligamentum patellae, cranial cruciate ligament, TTA

Cranial cruciate ligament (ligamentum cruciatum craniale, $\mathrm{LCCr}$ ) disease is one of the most common disorders of the knee joint in the dog (Pacchiana et al. 2003). Disturbed integrity of the LCCr causes translational and rotational instability of the knee joint (Arnoczky and Marshall 1977). There are many surgical procedures to secure the stability of the knee joint with disrupted cranial cruciate ligament. All these methods can be divided into static or dynamic in accordance to the technique used (Boudrieau 2009; Kühn et al. 2011). Tibial plateau levelling osteotomy (TPLO) and tibial tuberosity advancement (TTA) are the most popular methods for dynamic stabilization of the stifle (Slocum and Slocum 1993; Montavon et al. 2002; Boudrieau 2009). Some studies were published about the patellar desmopathy after the TPLO procedure (Pacchiana et al. 2003; Carey et al. 2005; Boudrieau 2009). However, one study deals with the changes within the patellar ligament after the TTA (Kühn et al. 2011).

The aim of this study was to determine the thickness of the patellar ligament in dogs after the TTA using computed radiography.

\section{Materials and Methods}

This retrospective study evaluated 20 knee joints of eighteen dogs treated surgically at the Department of Surgery and Orthopaedics, Small Animal Clinic, Faculty of Veterinary Medicine, University of Veterinary and Pharmaceutical Sciences Brno between April 2007 and September 2012. Inclusion criteria were surgically confirmed diagnosis of the LCCr rupture, series of radiographs of the affected knee joint and TTA stabilization of the affected knee joint. There were three radiographic examinations in each dog. The first radiographic examination was done right before the surgery (T0), the second examination in the $7^{\text {th }}$ week (T1) and the third one in the $15^{\text {th }}$ week (T2) after the surgery. 
The radiographic examination was done with the X-ray machine Proteus XR/a and cassettes for computed radiography. The digital radiographs were stored in the DICOM format using computed radiography system Capsula XL (Fuji, Japan). Resolution of the radiographs was $1770 \times 2370$ and $1576 \times 1976$, respectively. Two orthogonal views of the stifle joint were made using the table top technique. The mediolateral projection was used for the measuring of the patellar ligament thickness. The thickness was measured in three different predetermined points within the patellar ligament (Plate III, Fig. 1). The first point of measurement was located $1 \mathrm{~cm}$ distally to the distal patellar apex (PV1), the second was located in the middle of the patellar ligament (PV2) and the third point was located $1 \mathrm{~cm}$ proximally to the patellar ligament attachment (PV3). To avoid some discrepancies because of different size of joints, and to correct the magnification, the ratios of each particular thickness and the length of the patellar ligament were calculated. These ratios were used for all the calculations within this study. Medical records of all the included dogs were searched for some demographic data (age, sex, body weight, type of LCCr rupture, arthrotomy, cage size, meniscal injury, time between surgery and radiographic examination).

All the statistical analysis was done with commercial software Minitab (Minitab Inc., Coventry, UK). To compare the measurements between each control a Wilcoxon test for paired data was used. The effect of the categorical variables (sex, type of LCCr rupture, arthrotomy, cage size, meniscal injury, time between surgery and radiographic examination) or continuous variables (age, weight) on the measured values was analysed by multivariate ANOVA (MANOVA).

\section{Results}

All of the 20 knee joints were radiographed immediately before the surgery (T0). Not every dog passed both two control examinations (T1 and T2). Only fourteen radiographs were taken in the T1 control examination (42-83 days after surgery, median 52 days, 7 weeks) and nine radiographs were taken in the T2 control examination (88-143 days after surgery, median 108 days, 15 weeks). Radiographs of just 6 knee joints were taken in both T1 and T2 control examinations. Measurements for each point (PV1, PV2 and PV3) between all the radiographic examinations (T0, T1 and T2) were compared. Significant differences were found between all the points of measurement in T1 control $(P<0.05)$ compared to T0. There was only one significant difference, for point PV2 $(P=0.013)$, in the case of T2 versus T0 comparison. No significant differences were found between all the points of measurement in T2 versus T1 control $(P>0.05)$. Results analyzed by the Wilcoxon test are shown in Table 1.

Table 1. Results of Wilcoxon test for comparisons of the patellar ligament thickness in dogs between each of radiographic examinations.

\begin{tabular}{llcc}
\hline Comparison & PV1 & PV2 & PV3 \\
\hline T1 vs. T0 & $0.003^{*}$ & $0.003^{*}$ & $0.010^{*}$ \\
T2 vs. T0 & 0.076 & $0.013^{*}$ & 0.124 \\
T2 vs. T1 & 0.529 & 1.000 & 0.529 \\
\hline
\end{tabular}

$\mathrm{T} 0$ - radiographic examination right before the surgery, $\mathrm{T} 1$ - radiographic examination $7^{\text {th }}$ week after the surgery, $\mathrm{T} 2$ - radiographic examination $15^{\text {th }}$ week after the surgery, PV1, PV2, PV3 - points for measuring the patellar ligament thickness (the exact location is described within the text), ${ }^{*}$ indicates significant results $(P<0.05)$

The effects of all the demographic variables on the thickness of the patellar ligament (age, sex, body weight, type of LCCr rupture, arthrotomy, cage size, meniscal injury, time between surgery and radiographic examination) were assessed using the MANOVA tests. From all the above mentioned variables only the time between surgery and radiographic examination had the significant effect on the thickness of the patellar ligament. The rest of the variables (age, sex, body weight, type of LCCr rupture, arthrotomy, cage size, meniscal injury) did not significantly influence the patellar ligament thickness. 


\section{Discussion}

In comparison to analogue radiography, computed radiography provides better contrast with the same exposition parameters. Good visualization of the patellar ligament is supported also by its superficial position and the surrounding fat tissue. These features give perfect possibility to evaluate the patellar ligament in the dog using computed radiography examination. However, only the thickness of the ligament can be assessed by this way. Ultrasonographic examination gives the possibility to assess also the inner structure of the ligament, but the interpretation is more operator dependent than interpretation of the radiographs.

Thickening of the patellar tendon is one of the signs of patellar desmopathy. It was described as a postoperative complication according to the TPLO surgery (Pacchiana et al. 2003; Mattern et al. 2006; Boudrieau 2009). Boudrieau (2009) explains the thickening of the patellar tendon as a result of shortening the distance between the patellar tendon attachment and the eminentia intercondylaris. This shortening is due to tibial osteotomy during the TPLO surgery and results in short lever arm during movements within the knee joint (flexion and extension). Greater force is needed to do the knee joint extension after the TPLO and patellar ligament is then more stressed (Boudrieau 2009). The method used by the TTA procedure is different and the distance between patellar tendon attachment and the eminentia intercondylaris gets longer (Plate III, Fig. 2). This changes cause a bigger lever arm and lower force is needed for the knee joint extension, and so patellar ligament is less stressed (Boudrieau 2009). To the authors' knowledge, only one study dealing with the changes within the patellar ligament after the TTA procedure has been published. The study confirms the presence of patellar desmopathy after the TTA (Kühn et al. 2011). Results of our study show that severe thickening of the patellar ligament appears 7 weeks after the surgery (T1), and that decrease of the ligament thickness is evident at the time of the following re-check 15 weeks after the surgery (T2). However, the difference between the measurements in the T1 versus T2 controls was not significant.
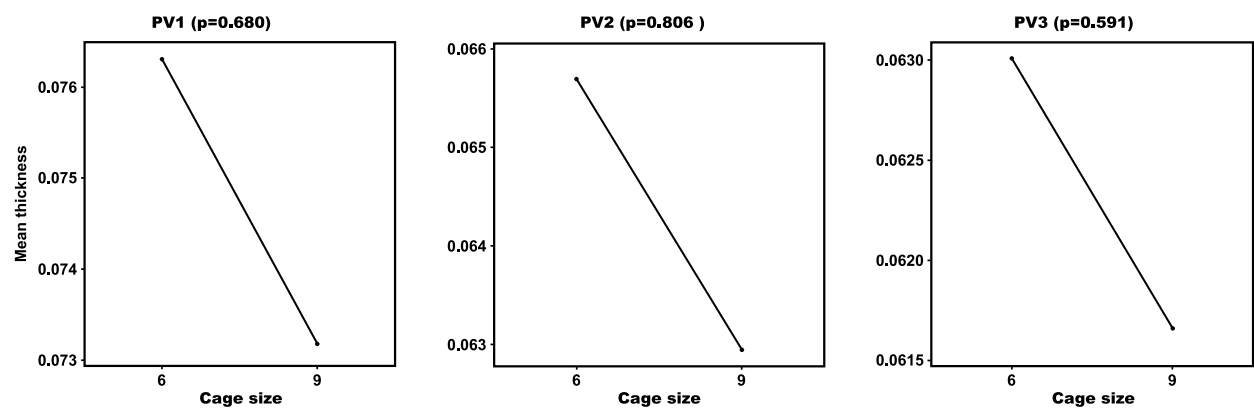

Fig. 3. The graphs show the effect of the size of the cage used for tibial advancement on the mean thickness of the patellar ligament in the dog. The patellar ligament thickness was smaller in each point of measurement when the bigger cage was used. The numbers in parentheses within the heading of each graph are probability values from the MANOVA analysis.

Kühn et al. (2011) stated that usage of a larger cage for tibial advancement caused bigger postoperative thickening of the patellar ligament. However, our results did not show any significant relationship between the size of the cage and the thickness of the patellar ligament. There is evidence of an opposite effect to the one stated by Kühn et al. (2011). Our results show that dogs with a bigger cage used for tibial advancement had 
smaller thickness of the patellar ligament (Fig. 3). However, this effect was considered non-significant for all the points of measurement $(P>0.05)$. The findings correlate with the theory published by Boudrieau (2009). The lack of significance in MANOVA could be a result of data analysis in a relatively small group of dogs used in the study. Future study in a larger group of clinically treated animals could extend our existing knowledge about different aspects related to the secondary patellar desmopathy in dogs after tibial osteotomies due to cranial cruciate ligament deficient knees. Another fact to consider is that not only the above mentioned factors themselves but also their interactions can have some effect on patellar ligament thickness.

\section{Acknowledgment}

This work was supported by the Ministry of Education, Youth and Sports intended for institutional research VFU Brno.

\section{References}

Arnoczky SP, Marshall JL 1977: The cruciate ligaments of the canine stifle: an anatomical and functional analysis. Am J Vet Res 38: 1807-1814

Boudrieau RJ 2009: Tibial plateau leveling osteotomy or tibial tuberosity advancement? Vet Surg 38: 1-22

Carey K, Aiken SW, DiResta GR, Herr LG, Monette S 2005: Radiographic and clinical changes of the patellar tendon after tibial plateau leveling osteotomy 94 cases (2000-2003). Vet Comp Orthop Traumatol 18: 235-242

Kühn K, Ohlerth S, Makara M, Hässig M, Guerrero TG 2011: Radiographic and ultrasonographic evaluation of the patellar ligament following tibial tuberosity advancement. Vet Radiol Ultrasound 52: 466-471

Mattern KL, Berry CR, Peck JN, Haan JJD 2006: Radiographic and ultrasonographic evaluation of the patellar ligament following tibial plateau leveling osteotomy. Vet Radiol Ultrasound 47: 185-191

Montavon PM, Damur D, Tepic S 2002: Advancement of the tibial tuberosity for the treatment of cranial cruciate deficient canine stifle In: 1st World Orthopaedic Veterinary Congress, Munich, Germany, September 2002, p. 152

Pacchiana PD, Morris E, Gillings SL, Jessen CR, Lipowitz AJ 2003: Surgical and postoperative complications associated with tibial plateau leveling osteotomy in dogs with cranial cruciate ligament rupture: 397 cases (1998-2001). J Am Vet Med Assoc 222: 184-193

Slocum B, Slocum TD 1993: Tibial plateau leveling osteotomy for repair of cranial cruciate ligament rupture in the canine. Vet Clin North Am Small Anim Pract 23: 777-795 
Plate III

Stehlík L. et al.: Radiographic... pp. 215-218

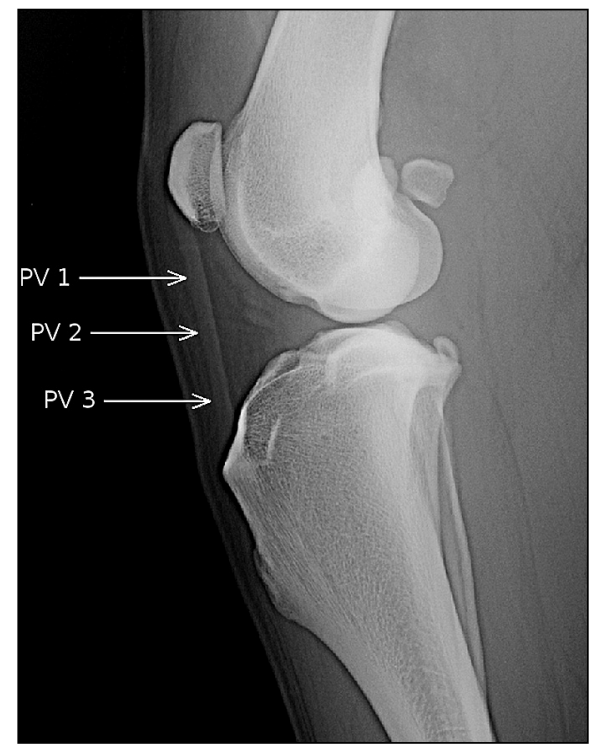

Fig. 1. Mediolateral radiograph of the cranial cruciate ligament deficient knee joint in dog right before the tibial tuberosity advancement surgery with three predetermined points of measurement on the patellar ligament.

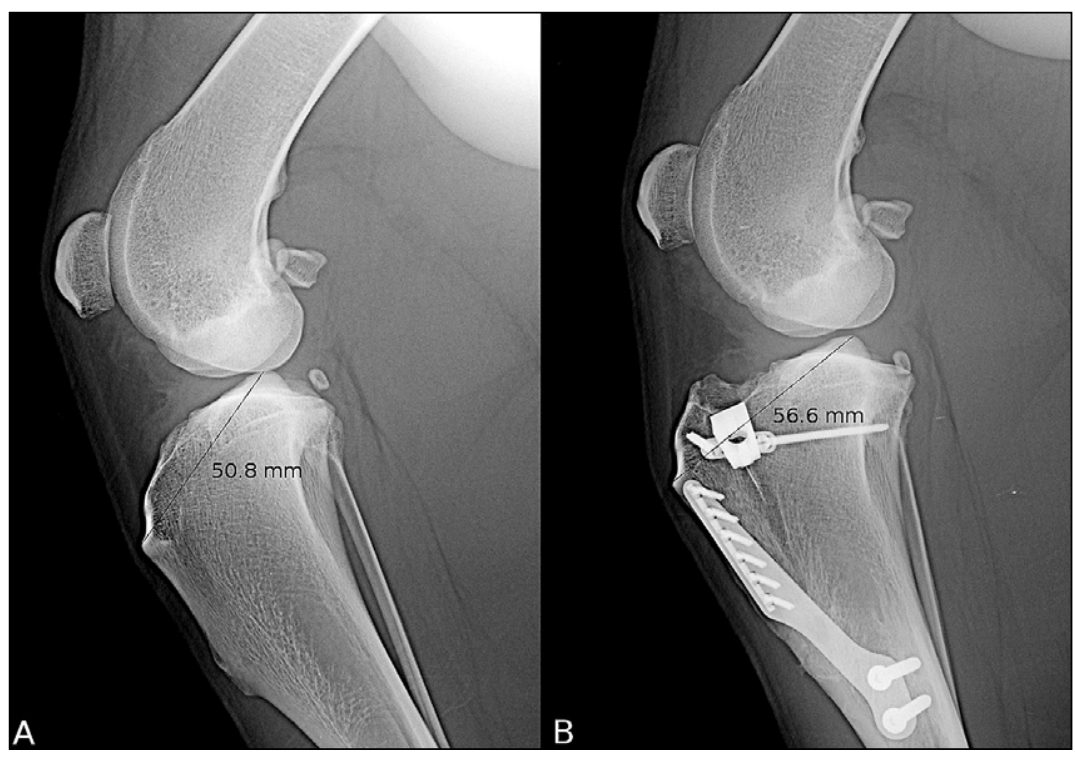

Fig. 2. Mediolateral radiographs of one knee joint of the dog right before the TTA surgery (A) and 7 weeks after the surgery (B). The distance between patellar tendon attachment and the eminentia intercondylaris is shown in the pictures. 